\title{
Do input modalities matter? Speed and reliability of touch inputs and mouse inputs in visual foraging
}

\author{
Marian Sauter ${ }^{1}$, Wolfgang Mack $^{1}$ \\ ${ }^{1}$ Bundeswehr University Munich, Institute of Psychology, General Psychology
}

\begin{abstract}
Visual attention is usually investigated in a variant of the visual search paradigm with a single search target to find in the scene. However, interactions with the natural environment often revolve around more than searching the environment and then executing a single decision. To alleviate these concerns, visual attention researches have recently started exploring the possibility of using touch-screen based foraging tasks. In these tasks, multiple targets have to be cancelled by tapping them. But the adoption remains poor, possibly because of the non-availability of large touch-screen devices in most research institutions. A viable substitute for cancelling targets in such search affordances could be the computer mouse. In the present study, we aimed at investigating how search performance differs in a visual foraging task depending on the input device (touch versus mouse). We presented a visual foraging task for a known target that had to be found multiple times per search display in several search conditions. We found that responses given via mouse are slightly slower in general, but the variances in responses are comparable in all measurements. We did not find disadvantages in using computer mouse in comparison to using a touch screen. We conclude that the computer mouse is an adequate alternative to touch devices and argue that using the computer mouse in visual attention studies should become better adopted.
\end{abstract}

Keywords: visual search; computer mouse; input device

Data, experiment and analyses scripts are available at

https://osf.io/8zbyf/

Author's note

There are no conflicts of interests to declare.

Correspondence concerning this article should be addressed to:

Marian Sauter, sauter.marian@gmail.com 


\section{Introduction}

A classical way of investigating visual attention involves visual search for single targets (Kristjánsson, 2006; Nakayama \& Martini, 2011; Wolfe, 2018). In fact, with the emergence of computers, almost all such visual search experiments were carried out by participants responding to simple stimuli appearing on the screen with the press of a key on the keyboard or a button box of some sort (Wolfe, 2018). This offers advantages as typically very fast and reliable responses can be achieved when the fingers already lay on or hover over the buttons. One limitation of this setup is that it biases task designs towards variants of the alternative (forced) choice (e.g. press 'left' when the target appears on the left side, press 'right' when the target appears on the right side) or go/no-go tasks (e.g. press 'up' when the target is present in the display, do not press when the target is absent). In addition, such a search in which a single decision is made within a given visual search scene might not be entirely realistic in applied scenarios. Interactions with the natural environment often revolve around more than searching the environment and then executing a single decision. To alleviate these concerns, visual attention researches have recently started exploring the possibility of using touch-screen based search task. Only in 2014, Kristjánsson, Jóhannesson, and Thornton introduced a 'finger foraging' paradigm, in which participants had to touch multiple targets on tablet devices in a visual search/foraging task. Although using fingers to directly touch the target seems intuitively plausible, one should also consider alternative input methods, such as the computer mouse. A group of researchers has introduced a software package to track mouse scan patterns (Freeman \& Ambady, 2010). Methodological research into using the mouse as an input device has revealed that there are other benefits as well. It was shown that there is a high correlation between mouse position and gaze position in website scanning tasks (Chen, Anderson, \& Sohn, 2001; Rodden, Fu, Aula, \& Spiro, 2008), offering an inexpensive, easy-to-setup way of getting an insight into participants' eye movements during search. It is possible to precisely characterize mouse trajectories' temporal and spatial dynamics, which can shed light on cognitive processes or be used as practical piloting run for eye tracking experiments. This cannot be done easily with touch responses, as only the actual response is logged, not the movement path of the hands. We would need additional tools, like video recording, to include movement patterns in the analysis.

Thus, the computer mouse could be an adequate alternative input device for research in visual search (and especially foraging) tasks, which can be used in a similar fashion like touch screens but can additionally offer insights from scan path analyses. It remains in question whether indicating responses with the finger on a touch screen offers any advantages over mouse responses. One thing that comes to mind is that touch presses are direct, so no stimulus-response mapping has to be learned by the participants and they do not need to control a hardware device (in a possibly unintuitive fashion) but simply use their finger. This could mean that the variance in response times is lower for touch responses compared to mouse responses. In the present experiments we aimed at investigating how search performance differs in a visual search task depending on the input device (touch versus mouse). We presented a common visual search task for a known target that had to be 
found multiple times per search display in several feature search and feature conjunction search conditions. We investigated whether there are benefits in terms of decreased response times (or variances in response times) emerging for one of the input devices and whether these possible benefits would represent (a) a general shift of response times, (b) are only prevalent in certain search conditions or (c) only affect the fastest or slowest response times.

Our objective was to investigate whether responses that are given via touch pad, prolong response times and lead to higher variance within a single participant and across participants. For this, we employed a foraging paradigm with blue and green objects. Participants were presented with search displays that either represented a feature search for color, size or shape or a feature conjunction search.

\section{Methods}

Participants. 25 participants (11 female, 14 male) took part in the experiment. All of them had normal or corrected-to-normal vision. Accurate color vision was ensured with a 10-plate Ishihara (1987) color test. They were naïve to the purpose of the study and provided written informed consent. All participants were students from the Bundeswehr University Munich.

Search displays. The search displays were assembled out of a combination of the objects presented in Figure 1 that were randomly arranged along an imaginary grid across the entire computer monitor. The sizes of the stimuli were 116x96 px (2x2 bricks), 156x108 px (2x3 bricks), 164x118 px (1x4 bricks) and 222x122 px (1x6 bricks). The individual bricks could never overlap each other. Participants always had to search for the green brick with six knobs (green-2x3). Either, all distractors were blue but could have different sizes and shapes

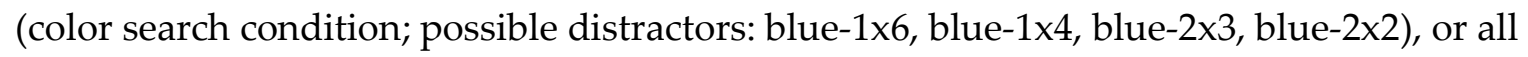
distractors were bricks with 4 knobs but could have different colors and shapes (size search

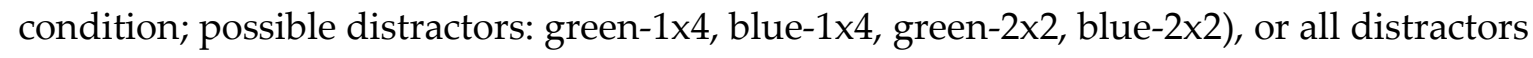
had the long shape but could have different sizes and colors (shape search condition;

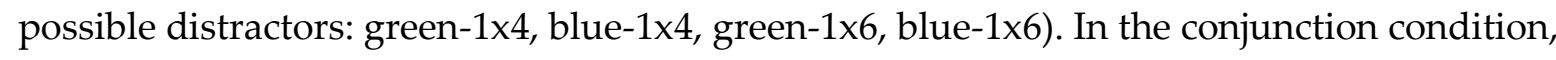
distractors would match the target in one feature but differ in another (conjunction condition). In any given display, there were one or two types of distractors, e.g. in one search

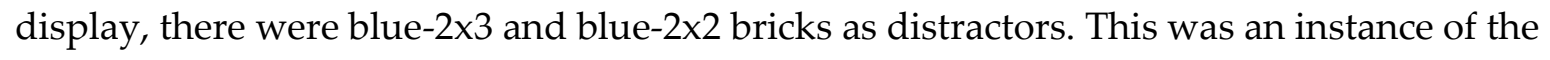
color search condition. In the conjunction condition this constraint led to five possible

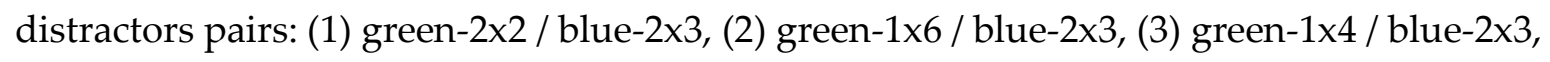

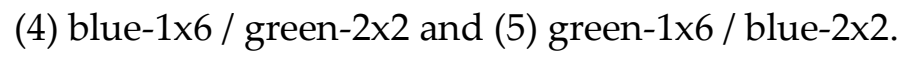

Overall, these combinations allowed for 20 different display setups (not accounting for differential item locations). In each display, there were four target items and 8, 16, 24 or 32 distractor items. Set sizes were presented randomly but all three set sizes were presented blocked for all of the 20 different display configurations. 


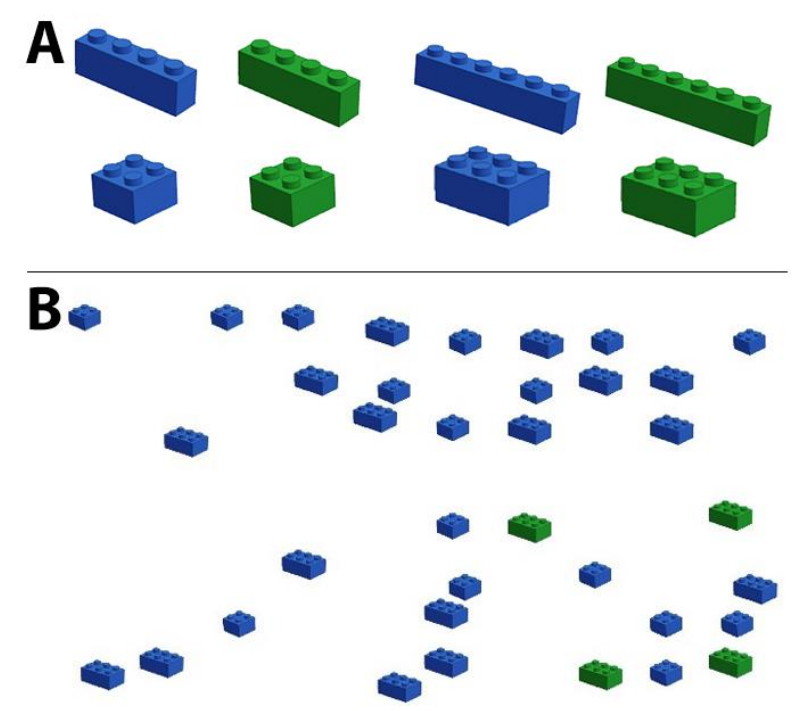

Figure 1. Overview of all employed stimuli (A) and an example of a search display (B).

Technical setup. Participants were placed in a sound-reduced test chamber. The search displays were presented on the same 23" HP EliteDisplay Business monitor in both conditions. Participants gave responses via touching the screen or by using standard twobutton Lenovo mouse in the mouse condition. Cursor sensitivity was set so that the whole display could be covered by moving the mouse a maximum of $10 \mathrm{~cm}$ on the mousepad. In both conditions, the resolution was set to $1920 \times 1080$ pixel @ 60Hz refresh rate. The experiment was programmed with OpenSesame 3.4.1. (Mathôt, Schreij, \& Theeuwes, 2012) using the Expyriment backend.

Procedure. Participants were given written and oral instructions. All participants completed both conditions (touch and mouse). They either started the experiment in the touch condition or the mouse condition (conditions blocked and counterbalanced). They were asked to tap on all target items (touch condition) or click on all target items (mouse condition) as fast and accurate as they could. For each of these conditions, they completed three blocks of 84 trials and received feedback on their average response times between the blocks. Errors were recorded when participants pressed on any distractor or the empty space but were not further analysed. In total, participants completed 504 trials and selected 2016 targets (four targets per display).

\section{Results}

We aimed to replicate the basic set size pattern (i.e. for hard searches, responses are slower when there are more distractors on the display). We expected that there are no set size effects for color searches but that there are set size effects for shape searches, size searches and conjunction searches. We submitted the mean RTs to repeated-measures ANOVA with the factors response condition (touch and mouse), search condition (size, shape, color and conjunction) and set size $(8,16,24$ and 32); for graphical representation, compare Figure 2. Again, all main effects were significant: input condition: $F(1,25)=99.07, p$ 
$<.001, \eta_{\mathrm{p}}{ }^{2}=0.8$; search type: $\mathrm{F}(3,75)=358.44, \mathrm{p}<.001, \eta_{\mathrm{p}}{ }^{2}=0.93$; and set size: $\mathrm{F}(3,75)=270.5$, $\mathrm{p}<.001, \eta_{\mathrm{p}}{ }^{2}=0.92$. The interaction of condition and search type was significant: $F(3,75)=$ 23.76, $\mathrm{p}<.001, \eta_{\mathrm{p}}{ }^{2}=0.49$; The interaction of condition and setsize was significant: $\mathrm{F}(3,75)=$ $3.66, \mathrm{p}=.016, \eta_{\mathrm{p}}{ }^{2}=0.13$; The interaction of search type and setsize was significant: $\mathrm{F}(9,225)=$ $57.49, \mathrm{p}<.001, \eta_{\mathrm{p}}{ }^{2}=0.7$; The interaction of condition and search type and setsize was significant: $F(9,225)=1.96, \mathrm{p}=.045, \eta_{\mathrm{p}}^{2}=0.07$.

To investigate for set size effects, we calculated a repeated measures ANOVA with the factor set size for each of the search conditions separately for touch and mouse response conditions. We first looked at the touch responses. For color searches, the set size main effect was significant, but is quite small, $\mathrm{F}(3,75)=3.43, \mathrm{p}=.021, \eta_{\mathrm{p}}{ }^{2}=0.12$. The set size effect was also significant for shape searches, $\mathrm{F}(3,75)=65.91, \mathrm{p}<.001, \eta_{\mathrm{p}}{ }^{2}=0.73$, for size searches, $\mathrm{F}(3$, $75)=116.11, \mathrm{p}<.001, \eta_{\mathrm{p}}{ }^{2}=0.82$ and for conjunction searches $\mathrm{F}(3,75)=91.43, \mathrm{p}<.001, \eta_{\mathrm{p}}{ }^{2}=$ 0.79 . The results for mouse responses show a different pattern as, for color searches, the set size effect was not significant, $\mathrm{F}(3,75)=0.67, \mathrm{p}=.572, \eta_{\mathrm{p}}^{2}=0.03$. But the set size effect was significant for shape searches: $F(3,75)=29.54, p<.001, \eta_{p}{ }^{2}=0.54$, for size searches: $F(3,75)=$ 84.46, $\mathrm{p}<.001, \eta_{\mathrm{p}}{ }^{2}=0.77$ and for conjunction searches: $\mathrm{F}(3,75)=79.98, \mathrm{p}<.001, \eta_{\mathrm{p}}{ }^{2}=0.76$.

A touch-response

set size 白 8 白 16 白 24 白 32

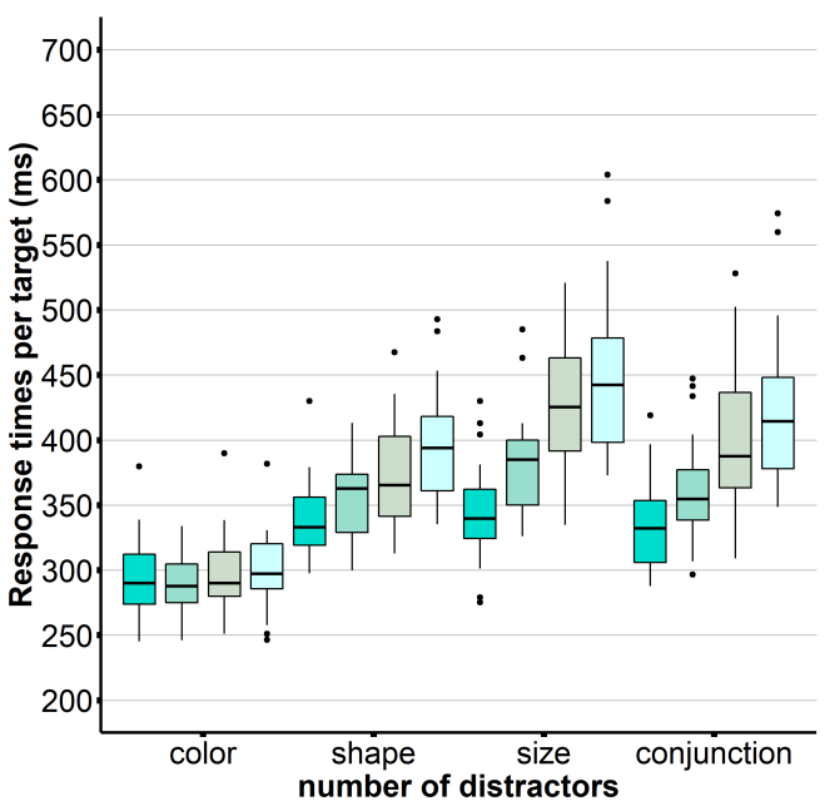

B mouse-response

set size 追 8 白 16 白 24 白 32

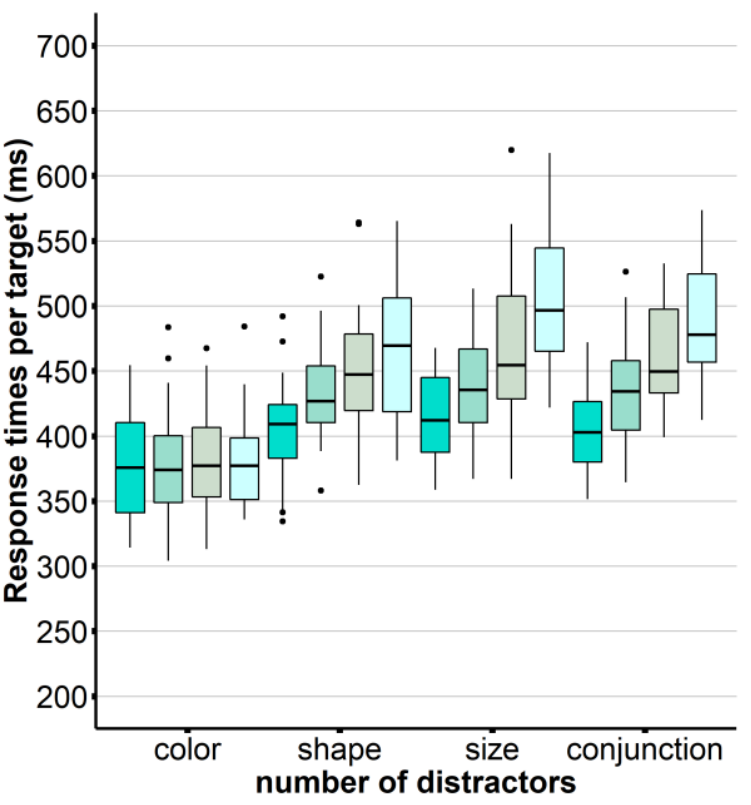

Figure 2. Response times as a function of the search condition for the touch condition (A) and the mouse condition (B). Colors indicate the set size. The lower and upper hinges (boxes) correspond to the first and third quartiles (the 25th and 75th percentiles). The upper whisker extends from the hinge to the largest value no further than $1.5^{*}$ IQR from the hinge (where IQR is the inter-quartile range, or distance between the first and third quartiles). The lower whisker extends from the hinge to the smallest value at most 1.5 * IQR of the hinge. Data beyond the end of the whiskers are outlying points and are plotted individually.

Then we contrasted the touch and mouse response times. When the responses were issued by touch compared to mouse, response times were still significantly faster, $383 \mathrm{~ms}$ vs $448 \mathrm{~ms} ; \mathrm{t}(49.22)=-6.39, \mathrm{p}<.001, \mathrm{~d}_{\mathrm{z}}=1.77,95 \% \mathrm{CI}[-86 \mathrm{~ms},-45 \mathrm{~ms}]$ across all conditions (compare Figure 2). We further visually explored the distribution of response times to see 
whether one of the input methods is imbalanced in terms of their response time distribution (Figure 3). In the touch condition (Figure 3A), we cannot observe a steep slope. Notably, the majority of the responses given are below $400 \mathrm{~ms}$ in all search conditions. This is unlike in the mouse condition (Figure 3B), in which we can see near-perfect linear distribution of response times in all conditions (aside from the ninth vincentile, which might be due to some extreme response times), but only in the color search condition, most of the responses are below $400 \mathrm{~ms}$. Overall, in both conditions, there is barely any difference between the three feature searches and conjunction searches - with the exception that there is a general shift in response times for color searches.

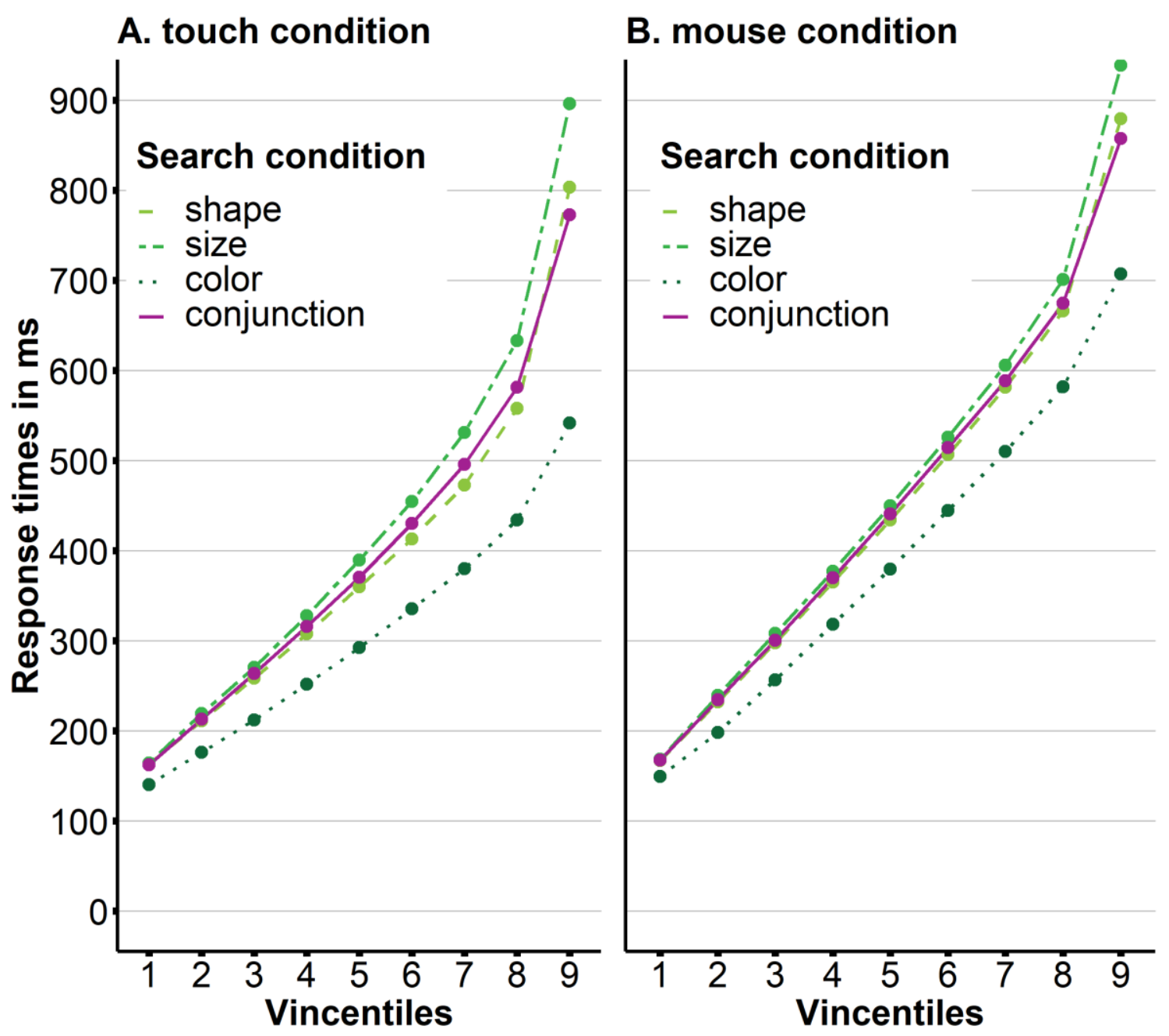

Figure 3. Response times as their function of the vincentiles plotted for the touch condition (A) and the mouse condition (B). Vincentiles are computed by sorting the response times and putting them into 9 equally-sized bins of which the mean is computed.

To compare the variances, we computed relative standard deviations (RSD) by multiplying the SD by 100 and dividing by the mean. The RSDs were not significantly different between touch and mouse responses, 49.52 vs $47.71 ; \mathrm{t}(48.19)=1.99, \mathrm{p}=.052, \mathrm{~d}_{\mathrm{z}}=$ $0.55,95 \% \mathrm{CI}[-0.02,3.60]$. Notably, the effect size is comparably big and the confidence interval only barely includes the zero value. To compare the variances more in detail, 
similarly to the RTs, we submitted the RSDs to a repeated-measures ANOVA with the factors input condition (touch and mouse), search condition (size, shape, color and conjunction) and set size $(8,16,24$ and 32) and concentrated on the input condition (c.f. Figure 4). This time, the main effect for input condition was significant, $F(1,25)=5.69, p=$ $.025, \eta_{\mathrm{p}}{ }^{2}=0.19$, as were the two-way interaction of input condition with search condition, $\mathrm{F}(3$, $75)=24.24, \mathrm{p}<.001, \eta_{\mathrm{p}}{ }^{2}=0.49$, the two-way interaction for input condition and set size, $\mathrm{F}(3$, $75)=5.82, p=.001, \eta_{p}^{2}=0.19$ and the three-way interaction with set size, $F(6,72)=2.04, p=$ $.071, \eta_{\mathrm{p}}{ }^{2}=0.15$. In subsequent Welch t-tests we contrasted touch and mouse responses for all set sizes and then all search types individually. For set sizes, we found that touch responses compared to mouse responses led to lower RSDs for set size $8,41.81$ vs $43.88, \mathrm{t}(43.28)=-2.91$, $\mathrm{p}=.006, \mathrm{~d}_{\mathrm{z}}=0.81,95 \% \mathrm{CI}[-3.50,-0.64]$ and higher variances for set sizes $16,46.13$ vs 45.07 ; $\mathrm{t}(39.92)=1.29, \mathrm{p}=.206, \mathrm{~d}_{\mathrm{z}}=0.36,95 \%$ CI [-0.61, 2.73], set size $24,50.05$ vs $47.11 ; \mathrm{t}(43.66)=$ $2.97, \mathrm{p}=.005, \mathrm{~d}_{\mathrm{z}}=0.82,95 \% \mathrm{CI}[0.94,4.93]$, and no difference for set size $32,53.23$ vs 50.87 ; $\mathrm{t}(46.86)=1.57, \mathrm{p}=.124, \mathrm{~d}_{\mathrm{z}}=0.43,95 \% \mathrm{CI}[-0.67,5.38]$. For search types, we found that touch responses compared to mouse responses led to lower variances for color searches, $39.45 \mathrm{vs}$ 43.72; $\mathrm{t}(37.44)=-7.32, \mathrm{p}<.001, \mathrm{~d}_{\mathrm{z}}=2.03,95 \% \mathrm{CI}[-5.45,-3.09]$ and there were no differences for size searches 51.29 vs $49.11 ; \mathrm{t}(48.05)=1.73, \mathrm{p}=.091, \mathrm{~d}_{\mathrm{z}}=0.48,95 \% \mathrm{CI}[-0.36,4.71]$, shape searches, 49.43 vs $47.84 ; \mathrm{t}(48.76)=1.17, \mathrm{p}=.248, \mathrm{~d}_{\mathrm{z}}=0.32,95 \% \mathrm{CI}[-1.14,4.32]$ or conjunction searches, 45.51 vs $45.63 ; \mathrm{t}(47.36)=-0.16, \mathrm{p}=.874, \mathrm{~d}_{\mathrm{z}}=0.04,95 \%$ CI $[-1.695,1.447]$.
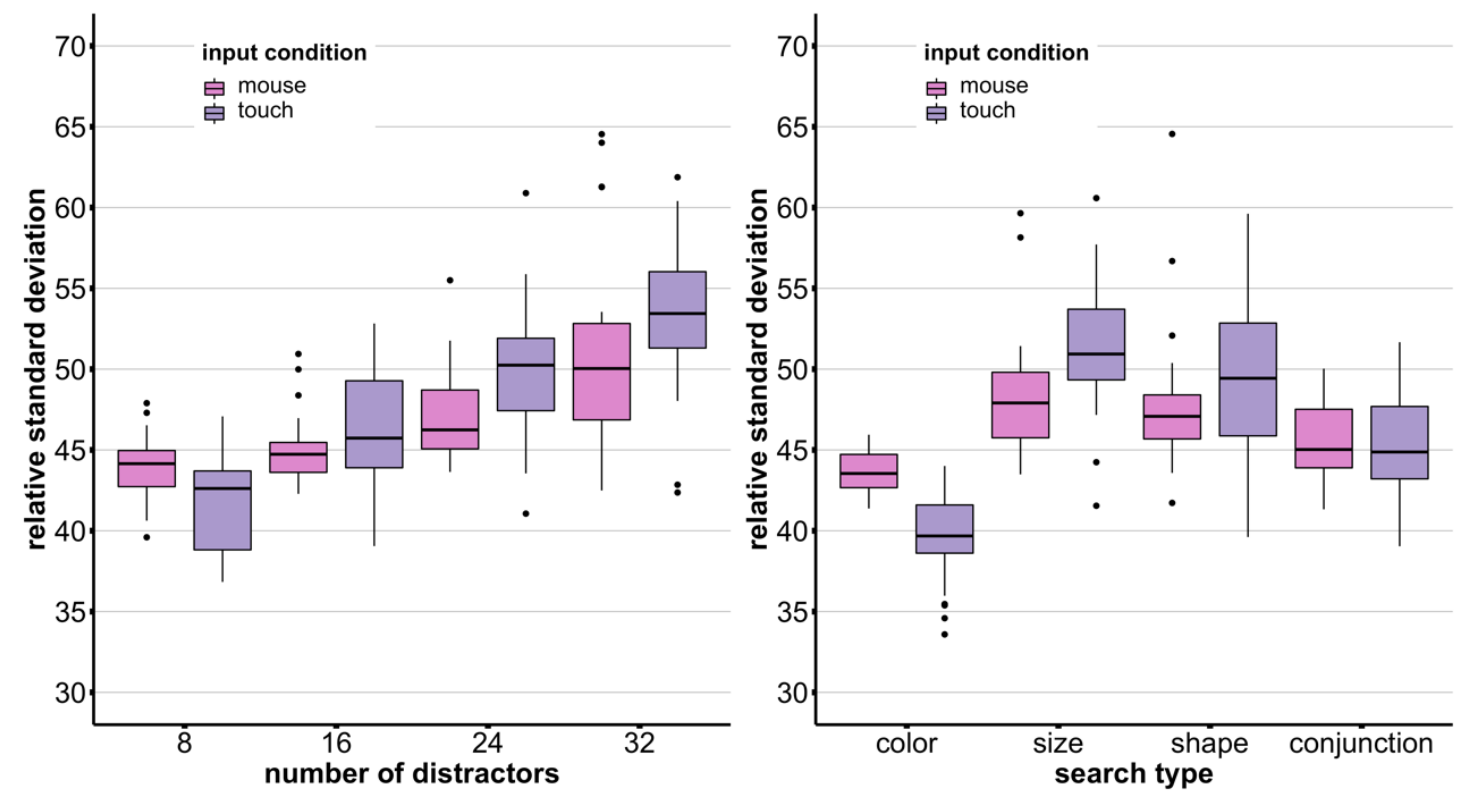

Figure 4. Relative standard deviations (RSD) as a function of the set size (left) and search type (right). Colors indicate the input condition. RSDs are computed per participant and condition by taking the standard deviations times 100 and dividing by their respective mean. The lower and upper hinges (boxes) correspond to the first and third quartiles (the 25th and 75th percentiles). The upper whisker extends from the hinge to the largest value no further than $1.5 *$ IQR from the hinge (where IQR is the inter-quartile range, or distance between the first and third quartiles). The lower whisker extends from the hinge to the smallest value at most 1.5 * IQR of the hinge. Data beyond the end of the whiskers are outlying points and are plotted individually. 


\section{Discussion}

In the present study, we investigated whether the computer mouse can be a viable alternative for giving responses in visual search or foraging tasks and compared it to inputs via touch screens. We found that generally, responses were given faster with touch inputs compared to mouse inputs ( $\sim 65 \mathrm{~ms})$. However, visually exploring the response time distributions, we found that there was a general shift towards faster responses for the color search condition across the whole RT distribution and this was true for both touch and mouse inputs. Interestingly, in the touch condition, all responses for color searches were given within $600 \mathrm{~ms}$ while the slowest responses in the mouse condition are above $700 \mathrm{~ms}$. It seems that the general RT difference between touch responses and mouse responses is not because most touch responses are given faster. It rather seems that only the slowest $10 \%$ of mouse responses are given slower on average compared to the touch responses, especially driven by the differences in the color condition.

From the variance analysis, it still seems that touch responses lead to less variable responses in the easiest (color) searches, arguing for employing touch devices whenever the search tasks are extremely easy. However, given these results we cannot give a general recommendation for touch devices as differences between the methods begin to fade when the search tasks get more difficult or set sizes increase beyond eight items. Numerically, it seems that, with increasing set sizes, variances for touch response increase steeper compared to mouse responses. It needs to be noted, that we did not exclude any outliers in our analyses and standard outlier rejection procedures might further align prior differences between the two input methods.

Recent research has shown that mouse scan paths are similar to scan paths gotten from eye tracking data (Chen et al., 2001; Rodden et al., 2008). Therefore, employing the mouse would lead to a simplistic way to design a pilot study for an eye tracking experiment. And while the correlation between mouse scan paths and eye scan paths in specific visual search paradigms still needs to be confirmed, there does not seem to be an inherent reason for not using the mouse as an input device. However, there is evidence that differing cognitive systems are at play when using touch input compared to mouse input. It was shown that while both eye and hand movements recruit visual attention at their respective target locations (eye: Deubel \& Schneider, 1996; Hoffman \& Subramaniam, 1995; Kowler, Anderson, Dosher, \& Blaser, 1995; hand: Deubel, Schneider, \& Paprotta, 1998; Rolfs, Lawrence, \& Carrasco, 2013), they recruit independent attentional resources (Jonikaitis \& Deubel, 2011) and their locations are represented on separate effector-specific maps (Hanning, Aagten-Murphy, \& Deubel, 2018). Thus, only if researchers are interested in these differing mechanisms between eye and hand movements in a visual search task, the mouse is not an adequate substitute for touch devices. From the input device perspective, they remain comparable.

Given our data, touch devices and the computer mouse seem comparable in terms of their reliability in visual search experiments and as the mouse was quite overlooked as an 
input devices, we argue that it should find larger adoption for multi-target searches or similar foraging studies. The final decision on what input device best suits one's needs should therefore be made according to which cognitive processes the researcher is interested in and what resources are available.

\section{References}

Chen, M. C., Anderson, J. R., \& Sohn, M. H. (2001). What can a mouse cursor tell us more?, 281. https://doi.org/10.1145/634067.634234

Deubel, H., \& Schneider, W. X. (1996). Saccade target selection and object recognition: Evidence for a common attentional mechanism. Vision Research, 36(12), 1827-1837. https://doi.org/10.1016/0042-6989(95)00294-4

Deubel, H., Schneider, W. X., \& Paprotta, I. (1998). Selective Dorsal and Ventral Processing: Evidence for a Common Attentional Mechanism in Reaching and Perception. Visual Cognition, 5(1-2), 81-107. https://doi.org/10.1080/713756776

Freeman, J. B., \& Ambady, N. (2010). MouseTracker: Software for studying real-time mental processing using a computer mouse-tracking method. Behavior Research Methods, 42(1), 226-241. https://doi.org/10.3758/brm.42.1.226

Geisler, W. S., Perry, J. S., \& Najemnik, J. (2006). Visual search: The role of peripheral information measured using gaze-contingent displays. Journal of Vision, 6(9), 1. https://doi.org/10.1167/6.9.1

Hanning, N. M., Aagten-Murphy, D., \& Deubel, H. (2018). Independent selection of eye and hand targets suggests effector-specific attentional mechanisms. Scientific Reports, 8(1), 9434. https://doi.org/10.1038/s41598-018-27723-4

Hoffman, J. E., \& Subramaniam, B. (1995). The role of visual attention in saccadic eye movements. Perception \& Psychophysics, 57(6), 787-795. https://doi.org/10.3758/bf03206794

Jonikaitis, D., \& Deubel, H. (2011). Independent allocation of attention to eye and hand targets in coordinated eye-hand movements. Psychological science, 22(3), 339-347. https://doi.org/10.1177/0956797610397666

Kowler, E., Anderson, E., Dosher, B., \& Blaser, E. (1995). The role of attention in the programming of saccades. Vision Research, 35(13), 1897-1916. https://doi.org/10.1016/00426989(94)00279-u

Kristjánsson, Á. (2006). Rapid learning in attention shifts: A review. Visual Cognition, 13(3), 324-362. https://doi.org/10.1080/13506280544000039 
Kristjánsson, Á., Jóhannesson, Ó. I., \& Thornton, I. M. (2014). Common Attentional Constraints in Visual Foraging. PLoS ONE, 9(6), e100752.

https://doi.org/10.1371/journal.pone.0100752

Mathôt, S., Schreij, D., \& Theeuwes, J. (2012). OpenSesame: an open-source, graphical experiment builder for the social sciences. Behavior Research Methods, 44(2), 314-324. https://doi.org/10.3758/s13428-011-0168-7

Nagy, A. L., Sanchez, R. R., \& Hughes, T. C. (1990). Visual search for color differences with foveal and peripheral vision. Journal of the Optical Society of America A, 7(10), 1995. https://doi.org/10.1364/josaa.7.001995

Nakayama, K., \& Martini, P. (2011). Situating visual search. Vision Research, 51(13), 15261537. https://doi.org/10.1016/j.visres.2010.09.003

Rodden, K., Fu, X., Aula, A., \& Spiro, I. (2008). Eye-mouse coordination patterns on web search results pages, 2997. https://doi.org/10.1145/1358628.1358797

Rolfs, M., Lawrence, B. M., \& Carrasco, M. (2013). Reach preparation enhances visual performance and appearance. Philosophical Transactions of the Royal Society B: Biological Sciences, 368(1628), 20130057. https://doi.org/10.1098/rstb.2013.0057

Wolfe, J. M. (2018). Visual Search, 1-55. https://doi.org/10.1002/9781119170174.epcn213 